\title{
Sustitución del pasto Megathyrsus maximus por guineo cuadrado y urea en mezclas ensiladas $^{1}$
}

\author{
Sustitution of Megathyrsus maximus grass by square guineo and urea in silage \\ mixtures
}

\author{
Michael López-Herrera², Augusto Rojas-Bourrillon², Ernesto Briceño-Arguedas ${ }^{3}$
}

1 Recibido: 20 de febrero, 2018. Aceptado: 13 de agosto, 2018. Este trabajo formó parte del proyecto de investigación 739-B4-115 inscrito en Vicerrectoría de Investigación, denominado "Evaluación de mezclas de pastos y forrajes con diferentes niveles de guineo cuadrado (Musa sp.) para la alimentación de rumiantes bajo normativa orgánica”. Universidad de Costa Rica, San José, Costa Rica.

2 Universidad de Costa Rica, Escuela de Zootecnia, Centro de Investigación en Nutrición Animal. San José, Costa Rica. michael.lopez@ucr.ac.cr (autor para correspondencia; https://orcid.org/0000-0003-4301-9900), augusto.rojas@ucr.ac.cr (https://orcid.org/0000-0002-9834-2361).

3 Finca Agroecológica Vocaré. Upala, Costa Rica. ebriceno@fincavocare.com

\section{Resumen}

Introducción. Los pastos tropicales poseen mayor contenido de fibra en proporción con otros nutrientes, esto limita el consumo de materia seca por parte de los rumiantes. El uso de aditivos en el ensilaje permite optimizar el contenido nutricional en los forrajes que pueden conservarse para utilizarlos en momentos de menor disponibilidad forrajera. Objetivo. El objetivo de esta investigación fue evaluar el efecto de sustituir en ensilados, porcentajes de pasto Megathyrsus maximus cv Mombaza por fruto inmaduro de guineo cuadrado (Musa ABB) y urea. Materiales y métodos. El experimento se desarrolló entre agosto y diciembre de 2016. Se utilizó un diseño factorial con ocho tratamientos. Las mezclas se almacenaron durante sesenta días en bolsas de 5,0 kg. Resultados. El uso de fruto de guineo cuadrado produjo incremento del $\mathrm{pH}$, el nitrógeno amoniacal, y la urea. El fruto también aumentó la concentración de materia seca y carbohidratos no fibrosos, esto mejoró el valor relativo del forraje y el contenido de energía. La urea mejoró en diez unidades porcentuales el contenido de proteína cruda, debido a su aporte de nitrógeno no proteico. El contenido de energía de los tratamientos varió entre 59,5 y 63,6\% TND, y la mayor energía se obtuvo en los tratamientos con mayor uso de fruto de guineo cuadrado. Conclusión. Los ensilados con 30\% de sustitución con fruto de guineo cuadrado y $0,5-1,0 \%$ de urea, se pueden considerar una alternativa para alimentación de rumiantes, como complemento de una dieta balanceada.

Palabras clave: conservación de forrajes, forrajes tropicales, aditivos para ensilaje, ensilaje.

\begin{abstract}
Introduction. Tropical pastures have higher fiber content in proportion to other nutrients, this limits the dry matter intake by ruminants. Usage of additives in silage can improve nutritive content in forages that can be preserved to use in moments of lower forage availability. Objective. The objective of this research was to evaluate the effect of substituting Megathyrsus maximus cv Mombaza grass for immature square bananas (Musa ABB) and urea in silages. Materials and methods. The experiment was carried out between August and December 2016. A factorial design with eight treatments was used. The forage mixtures were stored for sixty days in bags of $5.0 \mathrm{~kg}$ bags. Results. Usage of
\end{abstract}


square banana fruit produced an increase in $\mathrm{pH}$, ammonia nitrogen, and urea. The fruit also increased the concentration of dry matter and non-fibrous carbohydrates, this improved the relative value of the forage and the energy content. On the other hand, urea improved the crude protein content by 10 percentage units, due to its contribution as non-proteic nitrogen. The energy content of the treatments fluctuated between 59.5 and 63.6\% TND, in the treatments with greater use of square banana fruit. Conclusion. Silage with $30 \%$ of substitution by square banana fruit and $0.5-1.0 \%$ urea are considered an alternative for feeding ruminants, as a complement to a balanced diet.

Keywords: forage preservation, tropical forages, silage additives, silage.

\section{Introducción}

Los sistemas de producción de ganado bovino en el trópico se caracterizan por tener el pasto como fuente primaria de alimento (McDermott, 2010), lo cual representa ciertas limitaciones, teniendo en cuenta que dichas pasturas (C4) poseen un mayor contenido de fibra y lignina, lo cual está directamente relacionado con la capacidad de consumo y digestibilidad, y disminuyen de esta forma la disponibilidad de nutrientes al animal (Améndola et al., 2016).

La suplementación con alimentos bajos en fibra y lignina, además de altos en energía y otros nutrientes, permite el aumento en la densidad de nutrimentos de la dieta (Wadhwa y Bakshi, 2013), a su vez, aumenta el consumo y cantidad de materia digestible (Ramírez et al., 2002). Existe la necesidad de contar con disponibilidad de este tipo de alimentos de calidad para consumo animal, por lo que, es de suma importancia utilizar estrategias para su conservación.

El ensilaje es una técnica de conservación de forraje por vía húmeda, consiste en almacenar forrajes frescos en ausencia de oxígeno, ahí ocurren transformaciones químicas y físicas que definen su calidad (Hiriart, 2008). El ensilaje permite mantener la disponibilidad del componente forrajero durante la estación seca o lluviosa, condiciones que reducen el rendimiento por hectárea de las pasturas (López-Herrera et al., 2015). Además, de preservar desechos agrícolas altos en carbohidratos ( $>20 \%$ carbohidratos no fibrosos), como es el caso del fruto de pejibaye (Bactris gasipaes), pulpa de cítricos (Citrus sinensis), yuca (Manihot esculenta) y camote (Ipomoea batatas) (Gutiérrez et al., 2003).

Se han utilizado diversos aditivos para mejorar el proceso de ensilaje de subproductos agroindustriales, entre ellos: urea (López-Herrera et al., 2015), pollinaza (Gutiérrez et al., 2003; Arce et al., 2015), pulpa de cítricos deshidratada, melaza de caña de azúcar y heno (Yitbarek y Tamir, 2014). El uso de urea como aditivo, es una práctica común en la elaboración de ensilajes con bajo contenido de proteína, puesto que aumenta el contenido de este nutriente (Neumann et al., 2010) en forma de nitrógeno no proteico, y mejora la estabilidad aeróbica del ensilado (Borges et al., 2011). En Costa Rica, Arroyo et al. (2003) reportaron beneficios del uso de urea, como incrementos en el aporte de proteína cuando evaluaron ensilados de pulpa de pejibaye. El uso de aditivos depende de cada productor, por lo que, existen muchas variantes en las mezclas para ensilar, así se deben tener claros los objetivos antes de definir una mezcla para ensilaje (Mühlbach, 2005).

El objetivo de esta investigación fue evaluar el efecto de sustituir en ensilados, porcentajes de pasto Megathyrsus maximus cv Mombaza con frutos inmaduros de guineo cuadrado (Musa ABB) y urea. 


\section{Materiales y métodos}

Para el desarrollo del experimento se utilizaron frutos inmaduros de guineo cuadrado (Musa acuminata x balbisiana, Grupo ABB) y pasto Megathyrsus maximus (Jacq.) B. K. Simon \& S. W. L. Jacobs (=Panicum maximum Jacq.) cv Mombaza, cosechado a cincuenta días, cuya composición se indica en el Cuadro 1. Tanto el pasto como los otros materiales se obtuvieron en la Finca Agroecológica Siempreverde ubicada en Upala, Alajuela, Costa Rica. La zona se caracteriza por ser de alta humedad, con un promedio de precipitación de $2500 \mathrm{~mm} / \mathrm{año} \mathrm{y}$ una temperatura promedio de $25{ }^{\circ} \mathrm{C}$. Los frutos de guineo cuadrado que fueron utilizados estaban completamente llenos y engrosados, aunque inmaduros. Por otra parte, todos los análisis fueron realizados en el laboratorio de bromatología del Centro de Investigaciones en Nutrición Animal de la Universidad de Costa Rica.

Cuadro 1. Composición nutricional de frutos inmaduros de guineo cuadrado (Musa ABB) y del pasto Mombaza (Megathyrsus maximus cv Mombaza), utilizados para la elaboración de ensilados. San José, Costa Rica. 2017.

Table 1. Nutritional composition of immature fruits of square banana (Musa ABB) and Mombaza grass (Megathyrsus maximus cv Mombaza), used for silage elaboration. San Jose, Costa Rica. 2017.

\begin{tabular}{lcc}
\hline & Guineo cuadrado & Pasto Mombaza \\
\hline MS (\%) & 27,51 & 22,01 \\
PC (\% MS) & 3,70 & 10,20 \\
FDN (\% MS) & 9,30 & 70,10 \\
FDA (\% MS) & 6,20 & 36,50 \\
Lignina (\% MS) & 5,50 & 2,56 \\
Almidón total (\% MS) & 80,1 & ND* \\
CNF (\% MS) & 82,3 & 7,69 \\
NDT (\%) & 86,4 & 51,1 \\
\hline
\end{tabular}

Materia seca (MS), proteína cruda (PC), fibra en detergente neutro (FDN), fibra en detergente ácido (FDA), lignina (LIG) carbohidratos no fibrosos (CNF) y nutrimentos digestibles totales (NDT). * No detectable / Dry matter (DM), crude protein (CP), neutral detergent fiber (NDF), acid detergent fiber (ADF), lignin (LIG), non-fibrous carbohydrates (NFC) and total of digestible nutrients (TND). * Not detectable.

El experimento se desarrolló entre agosto y diciembre de 2016, y constó de un diseño factorial 3x2 completamente aleatorizado, donde se aplicaron dos niveles de fruto inmaduro de guineo cuadrado (15 y $30 \% \mathrm{p} / \mathrm{p}$ ) y tres niveles de urea $(0,0,5$ y $1,0 \%$ p/p). A todos los tratamientos se les agregó melaza ( $3 \%$ p/p) e inóculo bacterial artesanal $(1 \mathrm{l} / \mathrm{t})$, preparado fermentado con melaza, suero de leche y microorganismos. Cada tratamiento se repitió cuatro veces para un total de veinticuatro silos de bolsa.

Para la conservación del material, se utilizaron bolsas de polietileno para empaque al vacío con capacidad para $5,0 \mathrm{~kg}$ y con un grosor de $0,0063 \mathrm{~mm}$. Cada bolsa se consideró como una unidad experimental. Todos los materiales se picaron con una picadora eléctrica hasta obtener un tamaño de partícula entre 3,0 y 5,0 cm. Los silos se mantuvieron en condiciones ambientales (temperatura de $25{ }^{\circ} \mathrm{C}$ y humedad relativa $>80 \%$ ) por sesenta días. Posterior a este periodo, se abrieron las bolsas y se muestreó con una sonda de PVC al interior de la bolsa para tomar la muestra que se analizó en el laboratorio. 
A todos los tratamientos se les realizaron análisis de nitrógeno amoniacal, mediante la metodología empleada por Tobía (2004), el pH se midió con un potenciómetro de hidrógeno. Las características organolépticas se determinaron con base en una escala donde el valor 1 es la mejor calificación, y conforme aumenta el valor en la escala significa una menor calificación. Para el olor se utilizó la siguiente escala: 1=olor láctico, 2=olor acético leve, $3=$ =olor acético fuerte y $4=$ =olor butírico. Mientras que el color se calificó como: $1=$ verde olivo, $2=v e r d e$ oscuro, $3=$ pardo y $4=$ negro. Finalmente, para la textura se utilizó la siguiente clasificación: $1=$ consistente, $2=$ suave y $3=$ mucílago. Al final todas las escalas se sumaron y los mejores tratamientos fueron los más cercanos a 3 y los tratamientos con peores características fueron los más cercanos a 11.

Se determinó el contenido de materia seca (MS), proteína cruda (PC), extracto etéreo (EE) y cenizas de acuerdo con la AOAC (1998); los carbohidratos no fibrosos (CNF) se determinaron según la metodología del NRC (2001). La concentración de fibra en detergente neutro (FDN), fibra en detergente ácido (FDA), hemicelulosa (HEM) y lignina, se determinaron mediante las metodologías descritas por Van-Soest et al. (1991). Tambián se estimó el contenido de nutrimentos digestibles totales (NDT) con las ecuaciones descritas por Weiss (2004), y para estimar el fraccionamiento de la energía se utilizaron las ecuaciones del NRC (2001). La concentración de FDN indigestible se estimó a partir del contenido de lignina, de acuerdo con la ecuación descrita en el trabajo de Detmann et al. (2004). Se calculó el contenido de proteína ligada a la fibra en detergentes neutro y ácido, mediante las metodologías descritas en el trabajo de Licitra et al. (1996).

Para el análisis de la información, se utilizó la prueba de Kruskal Wallis para variables no paramétricas como la calificación organoléptica; además, se utilizó análisis de varianza. Los resultados de ambas pruebas se obtuvieron a través del software INFOSTAT (Di-Rienzo et al., 2013), el análisis de la información se realizó de acuerdo con la siguiente ecuación:

$\mathrm{y}_{\mathrm{ijk}}=\mu+\mathrm{G}_{\mathrm{i}}+\mathrm{U}_{\mathrm{j}}+(\mathrm{GxU})_{\mathrm{ij}}+\varepsilon_{\mathrm{ijk}}$

Donde:

y = es la variable de respuesta obtenida de la ecuación.

$\mu=$ es la media general.

$\mathrm{U}=$ es el efecto $i$ ésimo de la urea en los tratamientos.

$\mathrm{G}=$ es el efecto $j$ ésimo del guineo cuadrado en los tratamientos.

$\mathrm{GxU}=$ es el efecto $i j$ ésimo de la interacción entre los aditivos urea y guineo cuadrado.

$\varepsilon=$ término de error, donde $\varepsilon \sim \mathrm{N}\left(0, \sigma^{2}\right)$.

Cuando se determinó significancia de los efectos principales, la comparación entre medias se realizó mediante la prueba de Tukey con un nivel de significancia de $\mathrm{p}<0,05$.

\section{Resultados}

\section{Parámetros de fermentación de los ensilados y calidad organoléptica}

El grado de acidez de los materiales ensilados se afectó de manera significativa por el uso de la urea $(\mathrm{p}=0,011)$ y del guineo cuadrado $(\mathrm{p}=0,029)$ en la mezcla ensilada. Conforme se incrementó la cantidad de guineo cuadrado en la mezcla, aumentó el potencial de hidrógeno de los ensilados; los tratamientos con 30\% de inclusión de guineo presentaron en promedio un valor de $\mathrm{pH}$ de 5,16 , mientras que, los tratamientos con $15 \%$ de fruto mostraron un valor promedio de 4,76 (Cuadro 2). 
Cuadro 2. Valores de $\mathrm{pH}$, nitrógeno amoniacal $\left(\mathrm{NH}_{3} / \mathrm{NT}\right)$ y materia seca (MS) de ensilados después de 60 días de fermentación, con sustitución de pasto Megathyrsus maximus cv Mombaza por fruto inmaduro de guineo cuadrado (Musa ABB) y urea. Centro de Investigación en Nutrición Animal, San José, Costa Rica. 2017.

Table 2. $\mathrm{pH}$ values, ammonia nitrogen $\left(\mathrm{NH}_{3} / \mathrm{NT}\right)$ and dry matter (MS) of silage after 60 days of fermentation, with substitution of Megathyrsus maximus cv Mombaza grass by immature fruits of square banana (Musa ABB) and urea. Animal Nutrition Research Center, San José, Costa Rica. 2017.

\begin{tabular}{|c|c|c|c|c|c|}
\hline Guineo cuadrado & Urea & pH & NH3/NT (\%) & MS (\%) & C.O.* \\
\hline \multirow[t]{3}{*}{15} & $\mathbf{0}$ & $4,5 \pm 0,2$ & $4,7 \pm 1,6^{\mathrm{a}}$ & $20,2 \pm 0,6^{\mathrm{a}}$ & $3,0^{\mathrm{a}}$ \\
\hline & 0,5 & $4,6 \pm 0,2$ & $6,7 \pm 0,8^{\mathrm{b}}$ & $19,6 \pm 0,7^{\mathrm{a}}$ & $5,3^{\mathrm{b}}$ \\
\hline & 1,0 & $5,2 \pm 0,3$ & $7,3 \pm 1,5^{\mathrm{b}}$ & $20,1 \pm 0,7^{\mathrm{a}}$ & $5,3^{\mathrm{b}}$ \\
\hline \multirow[t]{3}{*}{30} & $\mathbf{0}$ & $4,9 \pm 0,6$ & $4,3 \pm 0,9^{\mathrm{a}}$ & $22,2 \pm 2,1^{\mathrm{b}}$ & $3,8^{\mathrm{a}}$ \\
\hline & 0,5 & $5,1 \pm 0,4$ & $8,2 \pm 0,8^{\mathrm{b}}$ & $22,0 \pm 1,0^{\mathrm{b}}$ & $5,3^{\mathrm{b}}$ \\
\hline & 1,0 & $5,5 \pm 0,5$ & $9,3 \pm 1,2^{\mathrm{b}}$ & $23,3 \pm 1,2^{\mathrm{b}}$ & $5,5^{\mathrm{b}}$ \\
\hline
\end{tabular}

* Calificación organoléptica / Organoleptic classification.

Letras distintas en la misma columna son diferentes $(\mathrm{p}<0,05)$ / Different letters in the same column are different $(\mathrm{p}<0.05)$.

La incorporación de urea en la mezcla ensilada mostró que los valores del potencial de hidrógeno aumentaron de manera proporcional a su incorporación. Se estimó una correlación media entre la cantidad de proteína que provino de la urea $(\mathrm{Q}=0,52)$; así, los tratamientos donde no se utilizó fueron los de menor valor de $\mathrm{pH}(4,70)$, mientras que los de mayor inclusión, presentaron en promedio un $\mathrm{pH}$ de 5,35. La concentración de nitrógeno amoniacal se afectó de manera significativa por la incorporación de urea $(\mathrm{p}<0,0001)$ y de guineo cuadrado $(\mathrm{p}=0,042)$ (Cuadro 2$)$. Los tratamientos en los que se sustituyó el pasto por $30 \%$ de guineo cuadrado, presentaron en promedio un punto porcentual más de nitrógeno amoniacal que los tratamientos con 15\%. Los tratamientos con 30\% de inclusión de guineo cuadrado presentaron en promedio $2,55 \%$ más de materia seca con respecto a los tratamientos con $15 \%$; sin embargo, no hubo diferencias entre ellos. También, conforme se incrementó la cantidad de proteína cruda que provenía de la urea, se incrementó el contenido de nitrógeno amoniacal al final del proceso de ensilaje.

No se encontraron diferencias entre los tratamientos donde se utilizó urea, se determinó una alta correlación $(\varrho=0,74)$ entre el valor de proteína cruda que provenía de la urea y la concentración de nitrógeno amoniacal en el silo. Además, se encontró que la incorporación de urea en la mezcla ensilada, afectó de manera significativa $(\mathrm{p}=0,0007)$ las características organolépticas de los tratamientos, pues aquellos sin inclusión de urea presentaron mejores características de color, olor y textura (Cuadro 2).

El contenido de MS de los tratamientos se vio afectado de manera significativa $(\mathrm{p}<0,0001)$ por la inclusión de guineo cuadrado, debido a que posee mayor contenido de materia seca que el pasto Mombaza (Cuadro 1), lo que incrementó el contenido de nutrimentos en la mezcla ensilada. En promedio los tratamientos con 30\% de inclusión de guineo cuadrado poseían 2,55\% más de materia seca con respecto a los tratamientos con $15 \%$ (Cuadro 2).

\section{Componentes celulares de los ensilados}

La concentración de proteína cruda de los ensilados fue afectada $(\mathrm{p}<0,05)$. La inclusión de urea y de guineo cuadrado afectó la concentración de proteína cruda de los ensilados $(\mathrm{p}<0,05)$. Los tratamientos que contenían mayor cantidad de fruto presentaron menor concentración de proteína cruda, debido a que el fruto posee un bajo contenido de esta fracción, lo que afectó de manera negativa la concentración de proteína. En promedio, los tratamientos con 15\% de inclusión de guineo cuadrado presentaron 5,3\% más de PC con respecto al promedio de los tratamientos con $30 \%$ de fruto (Cuadro 3). 
Cuadro 3. Concentraciones de proteína cruda (PC), proteína insoluble en detergente neutro (PIDN), proteína insoluble en detergente ácido (PIDA), extracto etéreo (EE), cenizas y carbohidratos no fibrosos (CNF) en base seca, de los ensilados con sustitución de pasto Megathyrsus maximus cv Mombaza por fruto inmaduro de guineo cuadrado (Musa ABB) y urea posterior a 60 días de fermentación. Centro de Investigación en Nutrición Animal, San José, Costa Rica. 2017.

Table 3. Crude protein concentrations (CP), neutral detergent insoluble protein (NDIP), acid detergent insoluble protein (ADIP), ether extract (EE), ash and non-fibrous carbohydrates (NFC) dry matter basis, of silages with substitution of Megathyrsus maximus cv Mombaza grass by immature fruits of square banana (Musa ABB) and urea after 60 days of fermentation. Animal Nutrition Research Center, San José, Costa Rica. 2017.

\begin{tabular}{cccccccc}
\hline $\begin{array}{c}\text { Guineo } \\
\text { cuadrado }\end{array}$ & Urea & $\begin{array}{c}\text { PC } \\
(\% \mathbf{M S})\end{array}$ & $\begin{array}{c}\text { PIDN } \\
(\boldsymbol{\%} \mathbf{P C})\end{array}$ & $\begin{array}{c}\text { PIDA } \\
(\boldsymbol{\%} \mathbf{P C})\end{array}$ & $\begin{array}{c}\text { EE } \\
(\boldsymbol{\%} \mathbf{M S})\end{array}$ & $\begin{array}{c}\text { Cenizas } \\
(\boldsymbol{\%} \text { MS })\end{array}$ & $\begin{array}{c}\text { CNF } \\
(\% \mathbf{M S})\end{array}$ \\
\hline \multirow{2}{*}{$\mathbf{1 5}$} & $\mathbf{0}$ & $9,1 \pm 0,6^{\mathrm{a}}$ & $21,47^{\mathrm{b}}$ & $15,73^{\mathrm{b}}$ & $2,5 \pm 0,9$ & $11,6 \pm 0,5^{\mathrm{b}}$ & $19,2 \pm 2,8^{\mathrm{a}}$ \\
& $\mathbf{0 , 5}$ & $14,6 \pm 2,1^{\mathrm{b}}$ & $15,93^{\mathrm{a}}$ & $11,25^{\mathrm{a}}$ & $2,1 \pm 0,3$ & $12,3 \pm 1,5^{\mathrm{b}}$ & $19,5 \pm 6,3^{\mathrm{a}}$ \\
& $\mathbf{1 , 0}$ & $19,3 \pm 2,3^{\mathrm{c}}$ & $13,33^{\mathrm{a}}$ & $7,25^{\mathrm{a}}$ & $2,4 \pm 0,8$ & $12,1 \pm 1,8^{\mathrm{b}}$ & $17,7^{\mathrm{b}} \pm 1,9^{\mathrm{a}}$ \\
\hline \multirow{3}{*}{$\mathbf{3 0}$} & $\mathbf{0}$ & $8,3 \pm 0,3^{\mathrm{a}}$ & $27,35^{\mathrm{c}}$ & $22,65^{\mathrm{c}}$ & $2,3 \pm 0,3$ & $10,4 \pm 0,5^{\mathrm{a}}$ & $32,1 \pm 1,4^{\mathrm{b}}$ \\
& $\mathbf{0 , 5}$ & $13,5 \pm 0,7^{\mathrm{b}}$ & $25,50^{\mathrm{c}}$ & $19,12^{\mathrm{b}}$ & $2,5 \pm 0,2$ & $10,6 \pm 0,8^{\mathrm{a}}$ & $33,5 \pm 5,5^{\mathrm{b}}$ \\
& $\mathbf{1 , 0}$ & $18,8 \pm 1,2^{\mathrm{c}}$ & $23,71^{\mathrm{b}}$ & $15,54^{\mathrm{b}}$ & $2,2 \pm 0,2$ & $10,3 \pm 1,2^{\mathrm{a}}$ & $38,1 \pm 6,4^{\mathrm{b}}$ \\
\hline
\end{tabular}

* Valor corregido para eliminar el aporte de la urea a la proteína cruda / * Corrected value to eliminate urea contribution to crude protein.

Letras distintas en la misma columna son diferentes a nivel de $\mathrm{p}<0,05$ / Different letters in the same column are different $(\mathrm{p}<0.05)$.

Conforme aumentó la cantidad de urea en la mezcla ensilada, incrementó la concentración de proteína, así, el promedio de los tratamientos con $0,5 \%$ de urea presentaron $2,8 \%$ más proteína cruda que el promedio sin urea; mientras que, el promedio de los tratamientos con 1,0\% mostraron 6,1\% más de proteína cruda, que el promedio de los tratamientos sin urea (Cuadro 3); aunque este aporte a la proteína cruda proviene en forma de nitrógeno no proteico (NNP). En cuanto a la cantidad de nitrógeno ligado a la fibra, se detectó que la inclusión de fruto de guineo cuadrado aumentó en 8,62\% la concentración de proteínas ligadas a la fibra en detergente neutro y en 7,69\% las ligadas a la fibra en detergente ácido.

No se encontraron diferencias en el contenido de extracto etéreo de las mezclas ensiladas, por efecto del fruto de guineo cuadrado, ni por la inclusión de urea. Sin embargo, el contenido mineral de los tratamientos estuvo influenciado únicamente por la inclusión de guineo cuadrado, donde se obtuvieron diferencias $(\mathrm{p}<0,05)$ entre los promedios de cada nivel de inclusión. Los tratamientos donde se utilizó 15\% de fruto de guineo cuadrado tuvieron $1,55 \%$ más de cenizas con respecto a aquellos con $30 \%$ del fruto.

El contenido de carbohidratos no fibrosos presentó diferencias $(\mathrm{p}<0,05)$ al usar fruto de guineo cuadrado en las mezclas ensiladas (Cuadro 3), más no hubo efecto de la urea sobre esta fracción. Así, los tratamientos con 30\% de guineo cuadrado tuvieron en promedio mayor contenido de esta fracción, en comparación con los ensilados donde se utilizó $15 \%$ de fruto.

\section{Componentes de la pared celular de los ensilados}

Todos los componentes de la pared celular presentaron diferencias estadísticas $(\mathrm{p}<0,05)$, debidas a la inclusión de fruto de guineo cuadrado; mientras que la inclusión de urea no generó diferencias. Cuando se utilizó fruto de guineo cuadrado en la mezcla ensilada se redujo el contenido de fibra, aunque también aumentó la concentración de lignina (Cuadro 4). De esta manera, en promedio los ensilados donde se utilizó 30\% de fruto de guineo cuadrado poseían mayor potencial de consumo de materia seca ( $2,45 \%$ del peso vivo de los animales), mientras que el 
Cuadro 4. Concentraciones de los componentes de la fibra en base seca, de los ensilados con sustitución de porcentajes de pasto Megathyrsus maximus cv Mombaza por fruto inmaduro de guineo cuadrado (Musa ABB) y urea posterior a 60 días de fermentación. Centro de Investigación en Nutrición Animal, San José, Costa Rica. 2017.

Table 4. Fiber components concentrations on dry matter basis, of silages with substitution of Megathyrsus maximus cv Mombaza grass by immature fruits of square banana $(M u s a \mathrm{ABB})$ and urea after 60 days of fermentation. Animal Nutrition Research Center, San José, Costa Rica. 2017.

\begin{tabular}{cccccccc}
\hline $\begin{array}{c}\text { Guineo } \\
\text { cuadrado }\end{array}$ & Urea & $\begin{array}{c}\text { FDN } \\
(\% \mathbf{M S})\end{array}$ & $\begin{array}{c}\text { iFDN } \\
(\boldsymbol{\%} \mathbf{F D N})\end{array}$ & $\begin{array}{c}\text { FDA } \\
(\boldsymbol{\%} \mathbf{M S})\end{array}$ & $\begin{array}{c}\text { HEM } \\
(\boldsymbol{\%} \mathbf{M S})\end{array}$ & $\begin{array}{c}\text { LIG } \\
(\boldsymbol{\%} \text { MS })\end{array}$ & VRF \\
\hline \multirow{2}{*}{$\mathbf{1 5}$} & $\mathbf{0}$ & $59,5 \pm 2,1^{\mathrm{b}}$ & $15,7 \pm 1,0^{\mathrm{a}}$ & $37,6 \pm 2,8^{\mathrm{b}}$ & $21,9 \pm 3,5^{\mathrm{b}}$ & $3,9 \pm 0,2^{\mathrm{a}}$ & $93,3 \pm 4,7^{\mathrm{a}}$ \\
& $\mathbf{0 , 5}$ & $58,2 \pm 3,9^{\mathrm{b}}$ & $16,2 \pm 1,5^{\mathrm{a}}$ & $35,9 \pm 4,3^{\mathrm{b}}$ & $22,3 \pm 1,8^{\mathrm{b}}$ & $3,9 \pm 0,1^{\mathrm{a}}$ & $97,8 \pm 11,4^{\mathrm{a}}$ \\
& $\mathbf{1 , 0}$ & $58,6 \pm 1,7^{\mathrm{b}}$ & $15,1 \pm 2,8^{\mathrm{a}}$ & $37,6 \pm 3,7^{\mathrm{b}}$ & $20,9 \pm 3,7^{\mathrm{b}}$ & $3,7 \pm 0,6^{\mathrm{a}}$ & $94,8 \pm 5,9^{\mathrm{a}}$ \\
\hline \multirow{3}{*}{$\mathbf{3 0}$} & $\mathbf{0}$ & $49,2 \pm 1,2^{\mathrm{a}}$ & $26,2 \pm 5,5^{\mathrm{b}}$ & $33,1 \pm 2,0^{\mathrm{a}}$ & $16,1 \pm 1,5^{\mathrm{a}}$ & $5,4 \pm 0,9^{\mathrm{b}}$ & $119,6 \pm 5,5^{\mathrm{b}}$ \\
& $\mathbf{0 , 5}$ & $51,2 \pm 3,9^{\mathrm{a}}$ & $23,9 \pm 8,6^{\mathrm{b}}$ & $33,5 \pm 2,6^{\mathrm{a}}$ & $17,7 \pm 1,3^{\mathrm{a}}$ & $5,1 \pm 1,7^{\mathrm{b}}$ & $114,9 \pm 12,4^{\mathrm{b}}$ \\
& $\mathbf{1 , 0}$ & $49,3 \pm 4,9^{\mathrm{a}}$ & $27,8 \pm 7,3^{\mathrm{b}}$ & $32,5 \pm 3,7^{\mathrm{a}}$ & $16,8 \pm 1,4^{\mathrm{a}}$ & $5,6 \pm 0,9^{\mathrm{b}}$ & $121,3 \pm 18,9^{\mathrm{b}}$ \\
\hline
\end{tabular}

* Fibra en detergente neutro (FDN), fibra en detergente neutro indigestible (iFDN), fibra en detergente ácido (FDA), hemicelulosa (HEM), lignina (LIG) y valor relativo del forraje (VRF) / * Neutral detergent fiber (NDF), indigestible neutral detergent fiber (iNDF), acid detergent fiber (FDA), hemicellulose (HEM), lignin (LIG) and Relative Forage Value (RFV)

Letras distintas en la misma columna son diferentes $(\mathrm{p}<0,05) /$ Different letters in the same column are different $(\mathrm{p}<0.05)$.

promedio de los tratamientos con $15 \%$ fue menor (2,06\% del peso vivo de los animales); los valores obtenidos fueron diferentes estadísticamente $(\mathrm{p}<0,05)$, debido a la concentración de fibra en detergente neutro.

El incremento de la lignina provino de utilizar más fruto de guineo cuadrado dentro de la mezcla, ya que el contenido de lignina de estos fue mayor que el del pasto (Cuadro 1). En consecuencia, esto generó diferencias $(\mathrm{p}<0,05)$ en la concentración de fibra en detergente neutro indigestible, por el contenido de fruto de guineo cuadrado en los ensilados, así, el promedio de fibra indigestible en los tratamientos con $15 \%$ de fruto fueron menores que el promedio de concentración de fibra en los tratamientos con 30\%. También se encontró diferencias $(\mathrm{p}<0,05)$ en el contenido de hemicelulosa por efecto del fruto de guineo cuadrado, ya que se disminuyó el contenido de este componente conforme se aumentó la cantidad de fruto en la mezcla (Cuadro 4).

El uso del fruto de guineo cuadrado generó diferencias $(\mathrm{p}<0,05)$ sobre el valor relativo del forraje, mientras que la urea no tuvo ningún efecto de sobre este parámetro (Cuadro 4). De esta manera, los tratamientos donde se utilizó $30 \%$ de guineo cuadrado presentaron mejor valor relativo $(118,6)$ con respecto al promedio de los que contenían $15 \%$ de fruto $(95,3)$.

\section{Caracterización energética de los ensilados}

Todas las fracciones de la energía de los materiales ensilados estuvieron influenciadas por la cantidad de guineo cuadrado utilizada en la mezcla $(\mathrm{p}<0,05)$. Los tratamientos con $30 \%$ de fruto fueron los que contuvieron mayor concentración de energía (Cuadro 5), esto se debió a que el fruto aportó gran cantidad de energía, sobre todo en forma de almidón (Cuadro 1).

En la Figura 1, se compara el aporte de energía por kilogramo de materia seca de los ensilados analizados, con otros forrajes utilizados en condiciones tropicales, como es el caso de ensilados de maíz (Betancourt, 2004; Cubero 
Cuadro 5. Fracciones de la energía de los ensilados con sustitución de porcentajes de pasto Megathyrsus maximus cv Mombaza por fruto inmaduro de guineo cuadrado (Musa ABB) y urea, posterior a 60 días de fermentación. Centro de Investigación en Nutrición Animal, San José, Costa Rica. 2017.

Table 5. Energy fractions of silage with substitution of Megathyrsus maximus cv Mombaza grass for immature fruits of square banana (Musa ABB) and urea, after 60 days of fermentation. Animal Nutrition Research Center, San José, Costa Rica. 2017.

\begin{tabular}{ccccccc}
\hline $\begin{array}{c}\text { Guineo } \\
\text { cuadrado }\end{array}$ & Urea & NDT (\%) & ${ }^{\mathbf{1}_{\mathbf{E N}}}$ & ${ }_{\mathbf{L}} \mathbf{E N}_{\mathbf{L}} \mathbf{( 3 \mathbf { x } )}$ & ${ }^{\mathbf{E}_{\mathbf{E N}}}$ & ${ }_{\mathbf{G}}$ \\
\hline \multirow{3}{*}{$\mathbf{1 5}$} & $\mathbf{0}$ & $59,6 \pm 1,0^{\mathrm{a}}$ & $1,34 \pm 0,02^{\mathrm{a}}$ & $1,32 \pm 0,01^{\mathrm{a}}$ & $0,72 \pm 0,04^{\mathrm{a}}$ & $1,30 \pm 0,07^{\mathrm{a}}$ \\
& $\mathbf{0 , 5}$ & $60,9 \pm 1,4^{\mathrm{b}}$ & $1,37 \pm 0,12^{\mathrm{b}}$ & $1,34 \pm 0,10^{\mathrm{b}}$ & $0,76 \pm 0,05^{\mathrm{a}}$ & $1,34 \pm 0,12^{\mathrm{b}}$ \\
& $\mathbf{1 , 0}$ & $59,5 \pm 1,7^{\mathrm{a}}$ & $1,34 \pm 0,02^{\mathrm{a}}$ & $1,32 \pm 0,01^{\mathrm{a}}$ & $0,72 \pm 0,07^{\mathrm{a}}$ & $1,29 \pm 0,08^{\mathrm{a}}$ \\
\hline \multirow{3}{*}{$\mathbf{3 0}$} & $\mathbf{0}$ & $63,2 \pm 1,6^{\mathrm{b}}$ & $1,43 \pm 0,06^{\mathrm{b}}$ & $1,38 \pm 0,04^{\mathrm{b}}$ & $0,83 \pm 0,04^{\mathrm{b}}$ & $1,42 \pm 0,06^{\mathrm{b}}$ \\
& $\mathbf{0 , 5}$ & $62,8 \pm 1,2^{\mathrm{b}}$ & $1,43 \pm 0,07^{\mathrm{b}}$ & $1,38 \pm 0,03^{\mathrm{b}}$ & $0,82 \pm 0,04^{\mathrm{b}}$ & $1,40 \pm 0,05^{\mathrm{b}}$ \\
& $\mathbf{1 , 0}$ & $63,6 \pm 1,8^{\mathrm{b}}$ & $1,44 \pm 0,06^{\mathrm{b}}$ & $1,39 \pm 0,05^{\mathrm{b}}$ & $0,84 \pm 0,08^{\mathrm{b}}$ & $1,43 \pm 0,06^{\mathrm{b}}$ \\
\hline
\end{tabular}

${ }^{1}$ (Mcal/kg MS) / (Mcal/kg DM). Nutrimentos digestibles totales (NDT), energía neta de lactación (EN ${ }_{\mathrm{L}}$ ), energía neta de ganancia de peso $\left(\mathrm{EN}_{\mathrm{G}}\right)$, energía neta de mantenimiento $\left(\mathrm{EN}_{\mathrm{M}}\right)$ / Total digestible nutrients $(\mathrm{TDN})$, net energy of lactation $\left(\mathrm{EN}_{\mathrm{L}}\right)$, net energy of weight gain $\left(\mathrm{EN}_{\mathrm{G}}\right)$, net energy of maintenance $\left(\mathrm{EN}_{\mathrm{M}}\right)$.

Letras distintas en la misma columna son diferentes $(\mathrm{p}<0,05)$ / Different letters in the same column are different $(\mathrm{p}<0.05)$.

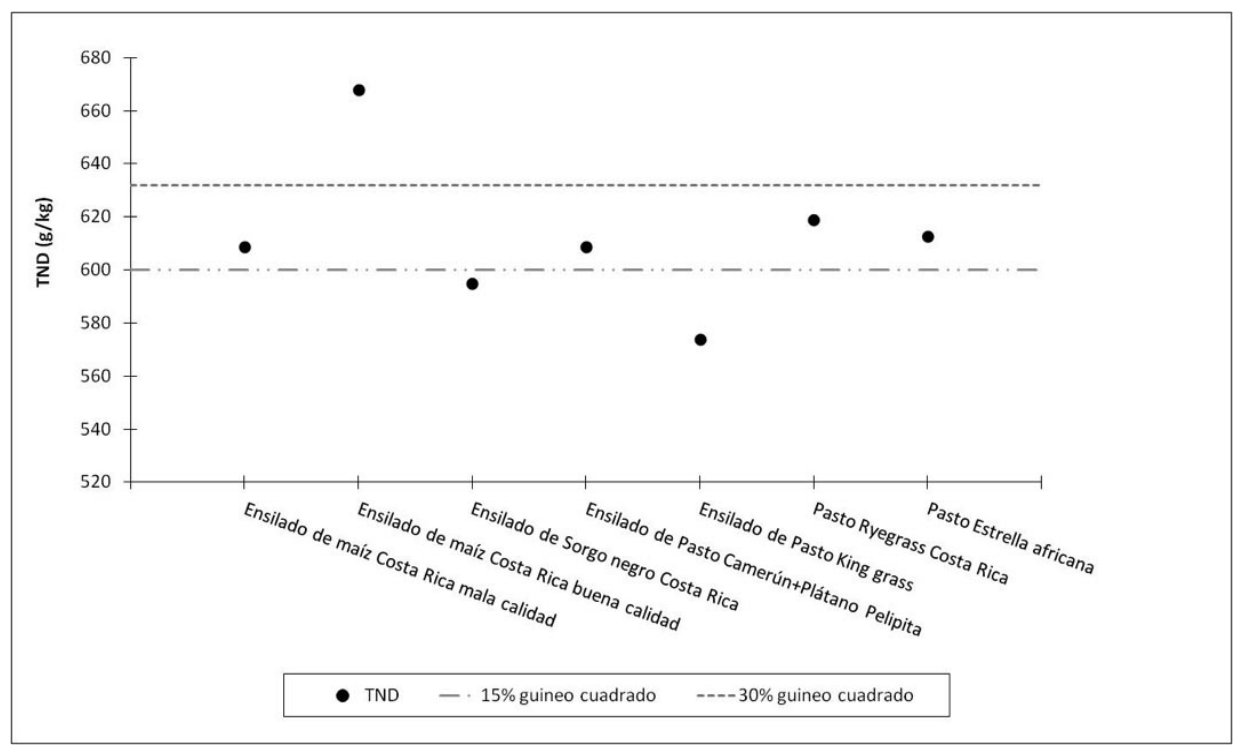

Figura 1. Cantidad de total de nutrientes digestibles (TND) ( $\mathrm{g} / \mathrm{kg} \mathrm{MS}$ ) de diferentes forrajes frescos y ensilados, en comparación con los tratamientos, de acuerdo al uso de fruto de guineo cuadrado (Musa ABB). San José, Costa Rica. 2017.

Figure 1. Total digestible nutrients quantity $(\mathrm{g} / \mathrm{kg} \mathrm{DM})$ from different fresh and silage forages, compared to treatments according to use of square banana fruit (Musa ABB). San José, Costa Rica. 2017.

et al., 2010), sorgo (Boschini-Figueroa y Elizondo-Salazar, 2005; Vargas-Rodríguez, 2005; Alpizar et al., 2014), pasto Camerún con 30\% plátano Pelipita (López-Herrera et al., 2017) y pastos frescos (Villalobos y Sánchez, 2010; Villalobos y Arce, 2014; Fukagawa et al., 2010). 


\section{Discusión}

\section{Parámetros de fermentación de los ensilados y calidad organoléptica}

Los valores de $\mathrm{pH}$ y nitrógeno amoniacal obtenidos en esta investigación estuvieron afectados por la cantidad de urea utilizada, este efecto ha sido evaluado en otras investigaciones donde se utilizaron otras fuentes de nitrógeno no proteico (Gutiérrez et al., 2003; Borges et al., 2011, Arce et al., 2015; López-Herrera et al., 2015).

El uso de fuentes con alta concentración de nitrógeno aumenta la capacidad amortiguadora de la mezcla forrajera (Giger-Reverdin et al., 2002), de esta manera se requiere más cantidad de carbohidratos solubles que permitan disminuir el potencial de hidrógeno en el silo (Kung y Shaver, 2001), para que sea posible la conservación apropiada del material, por esta razón, si no hay disponibilidad de carbohidratos solubles, el pH no disminuye lo necesario para evitar la proliferación de bacterias clostridiales en el silo, que consumen el ácido láctico y descomponen el forraje (Hiriart, 2008).

Aunque el guineo cuadrado posee un elevado contenido de carbohidratos no fibrosos (80,1\% MS), no hubo diferencias en el pH o en la concentración de nitrógeno amoniacal, entre los niveles de sustitución con fruto, esto sugiere que los carbohidratos de la melaza fueron consumidos y los del fruto estuvieron en forma poco disponible para las bacterias en el silo, lo que coincide con Barrera et al. (2010), quienes describieron que, en los frutos inmaduros, los carbohidratos dominantes están en forma de almidones y muy pocos azúcares solubles, lo que limita su acción en el ensilaje. Los almidones tienen muy poca participación en el proceso de ensilaje (López-Herrera y Briceño-Arguedas, 2017), ya que las enzimas capaces de degradarlos se inactivan en condiciones de acidez como las del ensilaje (Neureiter et al., 2005). Por lo tanto, se deben adicionar más carbohidratos solubles a la mezcla si se desea obtener un valor de pH menor a 4,50, indicado como ideal para ensilados estables (Kung y Shaver, 2001; Hiriart, 2008), esto se puede lograr a través del uso de melazas (Yitbarek y Tamir, 2014).

El contenido de humedad de los forrajes puede afectar el proceso fermentativo, materiales con mayor contenido de humedad tienden a estabilizarse a pH mayores que los ensilados más secos (McDonald, 1981; LópezHerrera y Briceño-Arguedas, 2017), tal y como ocurrió en este experimento (Cuadro 2). Este comportamiento fue determinado en el trabajo de López-Herrera et al. (2015), quienes encontraron valores de pH cada vez menores (de 7,1 a 4,3) conforme se adicionó heno de pasto Transvala (Digitaria decumbens, Stent., cv. Transvala) a ensilados de coronas de piña, esto debido al aumento en la concentración de materia seca que promueve el heno $(80,22 \pm 4,82 \%)$ (WingChing y Alvarado, 2009).

Los valores de $\mathrm{pH}$ en los materiales ensilados fueron mayores a los obtenidos por Betancourt $(2004)(3,55)$, Cubero et al. (2010) $(3,47$ - 3,67) en ensilados de maíz, López-Herrera et al. (2015) $(4,30$ - 7,10) en mezclas ensiladas de coronas de piña, heno, pulpa de cítricos deshidratada y urea, y que los publicados por Arce et al. (2015) $(4,30-4,63)$ en ensilados de subproductos de yuca con inclusión de pollinaza. Esto pudo deberse a un conjunto de factores tales como, contenido de humedad mayor al 75\% en el material forrajero (McDonald, 1981) (Cuadro 1) y bajo contenido de carbohidratos solubles en la mezcla ensilada (Kung y Shaver, 2001; Hiriart, 2008), también pudo ser consecuencia del incremento en la capacidad amortiguadora de la mezcla forrajera, debido a la adición de compuestos nitrogenados por medio de la urea (Giger-Riverdin et al., 2002).

Las diferencias de color provienen de la oxidación enzimática de los fenoles en el fruto de guineo cuadrado, lo cual promueve el pardeamiento del forraje (Quiceno et al., 2014), lo que excluye que haya habido cambios de coloración y pérdidas de calidad nutricional por elevación de la temperatura (Hiriart, 2008). El olor indica la calidad del proceso de fermentación en el silo, en este caso se encontró que los ensilados con mayor inclusión de urea presentaron mayor concentración de ácido acético, debido a una mayor capacidad amortiguadora, y el resto de las implicaciones consecuentes que también afectan el pH y el nitrógeno amoniacal (López-Herrera y BriceñoArguedas, 2017). Finalmente, la textura indica el grado de descomposición del material, es decir, qué tanto se han 
descompuesto las estructuras originales que componen la mezcla, también es afectada por la humedad en el silo y la oxidación del material (Hiriart, 2008).

El contenido de materia seca de los ensilados se incrementó debido a que el fruto de guineo cuadrado posee menor contenido de humedad, sin embargo, es posible que estas mezclas forrajeras hayan producido efluentes, ya que presentaron valores de materia seca inferiores al 35\% MS, deseable para un adecuado ensilaje (Hiriart, 2008), lo cual, no se puede constatar por la técnica de microsilos, lo que limita esta medición (Cherney et al., 2004; LópezHerrera et al., 2009).

\section{Componentes celulares de los forrajes ensilados}

El uso de urea en las mezclas ensiladas permitió incrementar el contenido de proteína cruda de los ensilados, debido a un elevado aporte de nitrógeno no proteico (Church et al., 2003). Este efecto ha sido encontrado en otras investigaciones donde se ha utilizado urea como aditivo para incrementar el porcentaje de proteína cruda (Gutiérrez et al., 2003; López-Herrera et al., 2015). En esta investigación, entre el 24 y $41 \%$ del aporte de proteína de las mezclas provino de nitrógeno no proteico, por lo que, se debe considerar su posible impacto en la concentración de nitrógeno ureico en leche (MUN, por sus siglas en inglés) y sangre (BUN, por sus siglas en inglés) de vacas lecheras si se utiliza en grandes cantidades, sin una adecuada cantidad de carbohidratos que permitan la asimilación del nitrógeno (Pardo et al., 2008).

Los valores de proteína obtenidos en esta investigación fueron mayores a los encontrados por Cubero et al. (2010) con ensilados de maíz, y que los resultados reportados en la investigación de Lima-Orozco et al. (2013) para ensilados de pasto guinea asociados con Dolichos sp. (10,1\% de la MS).

En cuanto a la proteína ligada a la fibra, los cambios en las concentraciones de estas fracciones en las mezclas pudieron ser debidas a dos factores principales, el primero relacionado con la concentración de estas fracciones en el fruto de guineo cuadrado, sin embargo, se requiere de mayor investigación para comprobar este punto; el segundo factor puede estar determinado por procesos de calor en el silo, provocados por mala compactación y bajo contenido de carbohidratos solubles durante el ensilaje (Adesogan, 2006).

Aunque el incremento en la cantidad del fruto aumentó la cantidad de proteína ligada a la fibra, el uso de urea redujo la proporción de proteína ligada en la mezcla ensilada, esto debido a que la urea incrementó la cantidad de proteína cruda, pero este aporte provino de NNP, lo que proporcionalmente redujo la representación de proteína insoluble en detergente neutro (PIDN) y en detergente ácido (PIDA) en el rubro de proteína cruda, de esta manera el NNP que provino de la urea representó hasta 53-56\% de la proteína cruda en los tratamientos con 1,0\% de urea.

El contenido de extracto etéreo de las mezclas ensiladas fue bajo ( $<10 \%$ de la MS), tal y como ocurre en dietas forrajeras, además, hay que valorar el contenido de ceras y esteroles de baja digestibilidad en esta fracción (Church et al., 2003); por lo que, no se puede considerar que realicen un aporte importante de energía. No se encontraron diferencias en el contenido de extracto etéreo de las mezclas ensiladas por efecto del fruto de guineo cuadrado, ni por la inclusión de urea, esto se debió a que ninguno de los materiales posee un contenido de lípidos totales que provoque un cambio en esta porción de la materia seca.

El uso de guineo cuadrado en los ensilados disminuyó la concentración de la fracción mineral, debido a un menor contenido de esta fracción en el fruto (Montero, 2016) (Cuadro 1). Esto es positivo, ya que las cenizas carecen de aporte energético en el rumen, por lo que al disminuir aumenta la cantidad de materia orgánica fermentable en el rumen que puede contribuir a la alimentación del rumiante (Hoffman, 2005).

Los valores de cenizas obtenidos en esta investigación fueron mayores a los publicados en el trabajo de López-Herrera et al. (2017) con ensilados de pasto Camerún y plátano Pelipita. Además, fueron mayores que los 
valores reportados por Gómez-Vázquez et al. (2011) con ensilados de caña de azúcar, urea y granos de maíz. Estas diferencias pudieron originarse por la especie del forraje, los aditivos utilizados en la mezcla ensilada y el contenido de cenizas en los frutos de guineo cuadrado, ya que cada forraje tiene un contenido de nutrimentos diferente de acuerdo con la especie y el manejo que recibe el cultivo, además, el contenido de nutrimentos varía de acuerdo con la especie de musácea utilizada (Pelissari et al., 2012).

El contenido de carbohidratos no fibrosos estuvo influenciado por el fruto de guineo cuadrado, ya que contenían cerca de $80 \%$ de almidón (Cuadro 1). La urea no afectó el cálculo de los carbohidratos no fibrosos, ya que su aporte es como nitrógeno no proteico, es decir, que al no ser fuente de proteína verdadera, su contribución no contabiliza en la ecuación, por lo que se elimina utilizando la ecuación descrita por Detmann y Valadares-Filho (2010).

Los valores de concentración de carbohidratos no fibrosos obtenidos en este experimento fueron mayores a los obtenidos por López-Herrera et al. (2017) con ensilados de pasto Camerún con 30\% de plátano Pelipita. Estas diferencias son debidas al contenido de esta fracción en cada uno de los forrajes utilizados.

El contenido de carbohidratos no fibrosos, almidón en particular, varía entre especies de musáceas y entre zonas geográficas (Mohapatra et al., 2010; Pelissari et al., 2012; Ravi y Mustaffa, 2013), esto obliga al análisis previo de los materiales que se van a utilizar para la elaboración de los ensilados. También fueron mayor a los resultados publicados por Cubero et al. (2010) (17,1-25,6\% de la MS) con ensilados de maíz; esta circunstancia provoca que el material deba ser balanceado en una dieta, para evitar que lleguen cantidades altas de almidón (>30\% MS) al rumen, y se generen efectos adversos, como acidosis ruminal (Zebeli et al., 2010).

\section{Componentes de la pared celular en los ensilados}

Todos los componentes de la pared celular presentaron diferencias estadísticas $(\mathrm{p}<0,05)$, debidas a la inclusión de fruto de guineo cuadrado; mientras que la inclusión de urea no generó diferencias. Cuando se utilizó fruto de guineo cuadrado en la mezcla ensilada, se redujo el contenido de fibra, aunque también aumentó la concentración de lignina (Cuadro 4). De acuerdo con Harper y McNeill (2015), existe una estrecha relación entre el contenido de fibra en detergente neutro y el consumo de alimento de los rumiantes, por lo que, cualquier reducción en esta fracción podría resultar en un incremento en el consumo voluntario de los animales, siempre y cuando la digestibilidad de la fibra sea aceptable. Lo anterior concuerda con lo encontrado en esta investigación, ya que, en promedio los ensilados donde se utilizó 30\% de fruto de guineo cuadrado poseían mayor potencial de consumo de materia seca (2,45\% del peso vivo de los animales), que los tratamientos con $15 \%$ ( $2,06 \%$ del peso vivo).

La reducción de la fibra en detergente neutro fue sobre todo en el contenido de hamicelulosa, ya que su concentración en los frutos de guineo cuadrado fue menor (3,1\% MS) en comparación con el pasto Mombaza (33,6\% MS). Este mismo efecto se obtuvo en el trabajo realizado por López-Herrera et al. (2017), aunque el contenido de hemicelulosa detectado por estos autores fue mayor que los tratamientos de la presente investigación.

El promedio de fibra en detergente neutro indigestible en los tratamientos con $15 \%$ de fruto fueron menores $(\mathrm{p}<0,05)$ que el promedio de concentración de fibra en los tratamientos con 30\%. Esto se debió al aumento en la cantidad de lignina en los ensilados (Cuadro 4), lo que concuerda con lo publicado por Jung (2012), quien señaló que incrementos en la concentración de lignina en ensilados, resulta en una disminución de la digestibilidad de la fibra del material forrajero.

El incremento de la lignina provino de utilizar más fruto de guineo cuadrado dentro de la mezcla, ya que el contenido de esta fracción en los frutos de guineo fue mayor que en el pasto (Cuadro 1). Otros autores señalan que la concentración de lignina en los frutos de las musáceas puede variar entre 6 y 17\% de la materia seca (Emaga et al., 2008; Mohapatra et al., 2010), por lo que, se debe analizar cada material para evitar impactos negativos sobre la digestibilidad de la fibra de los materiales forrajeros que servirán de alimento a los rumiantes. 
Aumentar la cantidad de guineo cuadrado en los ensilados disminuyó la digestibilidad de la fibra; pero la calidad de la mezcla total se incrementó por el aporte de carbohidratos no fibrosos a los ensilados, lo que mejoró el valor relativo del forraje, que en consecuencia mejoró el aporte de nutrimentos al animal (Ferreira et al., 2017).

El uso del fruto de guineo cuadrado fue el que generó diferencias estadísticas $(\mathrm{p}<0,05)$ sobre el valor relativo del forraje, mientras que la urea no tuvo ningún efecto de sobre este parámetro. Con base en lo anterior, los tratamientos con $15 \%$ de fruto de guineo cuadrado se calificaron como de grado 2, mientras que los tratamientos con 30\% de fruto la calificación es de grado 1, según la tabla expuesta en el trabajo de Texeira y Andrade (2001); esto los clasifica como recursos forrajeros de calidad, tal y como se comparó con otros ensilados y pastos publicados por Texeira y Andrade (2001) y Hancock (2011).

\section{Caracterización energética de los ensilados}

El fruto de guineo cuadrado incrementó el contenido energético de los ensilados, debido a que poseía $86 \%$ de TND (2,01 Mcal EN $\mathrm{L}_{\mathrm{L}} / \mathrm{kg} \mathrm{MS}$ ) (Cuadro 1), por lo tanto, a mayor inclusión del fruto mayor densidad energética tendrán los ensilados, por un aporte de almidón tal y como se mencionó anteriormente. Por lo tanto, el incremento en la densidad energética de los ensilados resultará en una mayor disponibilidad de energía para las bacterias del rumen lo que se traduce en mayor rendimiento productivo de los animales. La urea no ejerció efecto alguno sobre la energía, debido a que su aporte es en forma de nitrógeno no proteico, el cual no posee valor energético para el animal (Church et al., 2003).

Los ensilados con $30 \%$ de sustitución por fruto de guineo cuadrado poseían un contenido de energía mayores a los encontrados por Betancourt (2004) y Cubero et al. (2010) en ensilados de maíz; Boschini-Figueroa y ElizondoSalazar (2005), Vargas-Rodríguez (2005) y Alpizar et al. (2014) con ensilados de sorgo negro; López-Herrera et al. (2017) con ensilados de pasto Camerún con 30\% plátano Pelipita; Fukagawa et al. (2010) con ensilados de pasto king grass; además de los reportados por Villalobos y Sánchez (2010) y Villalobos y Arce (2014) en pastos frescos (Figura 1). De esta manera, se puede indicar que los ensilados obtenidos tienen el contenido de energía $\left(\mathrm{EN}_{\mathrm{L}}, \mathrm{EN}_{\mathrm{M}}\right.$ y $\mathrm{EN}_{\mathrm{G}}$ ) suficiente para ser validados como complemento alimenticio en dietas para rumiantes.

Los ensilados obtenidos en esta investigación proveyeron 0,27 Mcal $\mathrm{EN}_{\mathrm{L}}$ por cada $\mathrm{kg} \mathrm{MS}$ de ensilado cuando se utilizó 15\% de sustitución por fruto de guineo cuadrado, mientras que, los ensilados con 30\% sustitución por

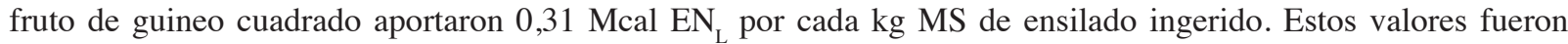
mayores a los obtenidos por Cubero et al. (2010) con ensilados de maíz (0,24 MCal), pero menores a los reportados por Alpízar et al. (2014) con sorgo forrajero (0,33 MCal).

Al utilizar el requerimiento para la producción de leche $\left(0,75 \mathrm{Mcal} \mathrm{EN}_{\mathrm{L}} / \mathrm{kg}\right.$ leche $4 \%$ grasa) de las tablas de requerimientos del NRC (2001), se determinó que un animal debe consumir 2,4 - 2,8 $\mathrm{kg}$ de ensilado para producir 1 $\mathrm{kg}$ de leche. Esto se debe principalmente al contenido de materia seca en cada forraje, lo cual disminuye la cantidad de energía contenida en cada kilogramo de forraje ingerido por el animal. Lo que refuerza la necesidad de mejorar los contenidos de materia seca de ensilados tropicales.

\section{Conclusiones}

La sustitución de pasto Mombaza por guineo cuadrado y urea, permitió una adecuada conservación del forraje y una mejora sustancial en el aporte de energía de la mezcla ensilada, cuando se utilizó 30\% de fruto en sustitución del pasto. Además, el contenido de proteína cruda mejoró al utilizar 0,5 - 1,0\% de urea en las mezclas ensiladas. Pero existió variación en el contenido de carbohidratos no fibrosos de los frutos de guineo cuadrado, lo que obliga al análisis previo antes de realizar el ensilaje. El uso de ambos aditivos mejoró la calidad nutricional y permitió 
elaborar ensilados que pueden ser incorporados en dietas para rumiantes, siempre y cuando se utilicen como complemento de una dieta balanceada. Sin embargo, se debe realizar investigación en cuanto al comportamiento ruminal de estos materiales y su impacto en sobre la productividad de rumiantes.

\section{Literatura citada}

Adesogan, A.T. 2006. Factors affecting corn silage quality in hot and humid climates. University of Florida, FL, USA. http:// dairy.ifas.ufl.edu/rns/2006/Adesogan.pdf (accessed Aug. 4, 2017).

Alpízar, A., M.I. Camacho, C. Sáenz, M.E. Campos, J. Arece, y M. Esperance. 2014. Efecto de la inclusión de diferentes niveles de morera (Morus alba) en la calidad nutricional de ensilajes de sorgo (Sorghum almum). Pastos y Forrajes 37:55-60.

Améndola, L., F.J. Solorio, J.C. Ku-Vera, R.D. Améndola-Massiotti, H. Zarza, and F. Galindo. 2016. Social behaviour of cattle in tropical silvopastoral and monoculture systems. Animal 10:863-867. doi:10.1017/S1751731115002475

AOAC (Association of Official Analytical Chemist). 1998. Official methods of analysis. 16 ${ }^{\text {th }}$ ed., $4^{\text {th }}$ rev. AOAC Int., Gaithersburg, MD, USA.

Arce, J., A. Rojas, y M. Poore. 2015. Efecto de la adición de pollinaza sobre las características nutricionales y fermentativas del ensilado de subproductos agroindustriales de yuca (Manihot esculenta). Agron. Costarricense 39:131-140.

Arroyo, C., A. Rojas-Bourrillon, y R. Rosales. 2003. Uso de urea o pollinaza como suplemento proteico para toretes consumiendo ensilaje de pulpa de pejibaye. Agron. Costarricense 27(2):69-73.

Barrera, J.L., G.S. Arrazola, y D.G. Cayón. 2010. Caracterización fisicoquímica y fisiológica del proceso de maduración de plátano Hartón (Musa AAB Simmonds) en dos sistemas de producción. Acta Agron. 59(1):20-29.

Betancourt, J.C. 2004. Caracterización nutricional y productiva de material fresco y ensilado de maní forrajero (Arachis pintoi) cultivado en asocio con maíz (Zea mays), a tres densidades de siembra. Tesis MSc., Universidad de Costa Rica, San José, CRC.

Borges J.A., Y. Bastardo, E. Sandoval, M. Barrios, y R. Ortega. 2011. Efecto de la adición de urea y el tipo de fermentación en la estabilidad de ensilajes de caña de azúcar (Saccharum spp.). Zootec. Trop. 29:283-291.

Boschini-Figueroa, C., y J. Elizondo-Salazar. 2005. Determinación de la calidad y la producción de sorgo negro forrajero (Sorghum almum) en edades para ensilar. Agron. Mesoam. 16:29-36. doi:10.15517/am.v16i1.11875

Cherney, D.J., J.H. Cherney, and W.J. Cox. 2004. Fermentation characteristics of corn forage ensiled in mini-silos. J. Dairy Sci. 87:4238-4246. doi:10.3168/jds.S0022-0302(04)73569-9

Church, D.C., W.G. Pond, y K.R. Pond. 2003. Fundamentos de nutrición y alimentación de animales. Limusa Wiley, México D.F., MEX.

Cubero, J.F., A. Rojas, y R. WingChing. 2010. Uso del inóculo microbial elaborado en finca en ensilaje de maíz (Zea mays). Valor nutricional y fermentativo. Agron. Costarricense 34:237-250.

Detmann, E., and S.C. Valadares-Filho. 2010. On the estimation of non-fibrous carbohydrates in feeds and diets. Arq. Bras. Med. Vet. Zootec. 62:980-984. doi:10.1590/S0102-09352010000400030

Detmann, E., J.T. Zervoudakis, L. da-Silva-Cabral, V.R. Rocha-Júnior, S.d.C. Valadares-Filho, A.C. de-Queiroz, N.J. Ponciano, e A.M. Fernandes. 2004. Validação de equações preditivas da fração indigestível da fibra em detergente neutro em gramíneas tropicais. Rev. Bras. Zootec. 33:1866-1875. doi:10.1590/S1516-35982004000700026

Agron. Mesoam. 30(1):179-194, enero-abril, 2019 
Di-Rienzo, J.A., F. Casanoves, M.G. Balzarini, L. Gonzalez., M. Tablada, y Y.C. Robledo. 2013. InfoStat versión 2013. Grupo InfoStat, FCA, Universidad Nacional de Córdoba, ARG.

Emaga, T.H., C. Robert, S.N. Ronkart, B. Wathelet, and M. Paquot. 2008. Dietary fibre components and pectin chemical features of peels during ripening in banana and plantain varieties. Bioresour. Technol. 99:4346-4354. doi:10.1016/j. biortech.2007.08.030

Ferreira, D.J., A.M. Zanine, R.P. Lana, A.L. Souza, M.D. Ribeiro, F.M. Negrão, G.R. Alves, and W.J.R. Castro. 2017. Intake and digestibility in sheep fed on marandu grass silages added with dehydrated barley. Pesq. Vet. Bras. 37(2):171-178. doi:10.1590/s0100-736x2017000200012

Fukagawa, S., J. Hirokawa, M. Okushi, and Y. Ishii. 2010. Fermentative quality and feed characteristics of dwarf napiergrass (Pennisetum purpureum Schumach) silage. Japan. J. Grass. Sci. 56(1):26-33. doi:10.14941/grass.56.26

Giger-Reverdin, S., C. Duvaux-Ponter, D. Sauvant, O. Martin, I. Nunes-do-Prado, and R. Müller. 2002. Intrinsic buffering capacity of feedstuffs. Anim. Feed Sci. Technol. 96:83-102. doi:10.1016/S0377-8401(01)00330-3

Gómez-Vázquez, A., J.M. Pinos-Rodríguez, J.C. García-López, E. de-la-Cruz-Lázaro, C. Luna-Palomera, and R. SánchezHernández. 2011. Nutritional value of sugarcane silage enriched with corn grain, urea, and minerals as feed supplement on growth performance of beef steers grazing stargrass. Trop. Anim. Health Prod. 43:215-220. doi:10.1007/s11250-0109678-Z

Gutiérrez, F., A. Rojas-Bourrillon, H. Dormond, M. Poore, y R. WingChing-Jones. 2003. Características nutricionales y fermentativas de mezclas ensiladas de desechos de piña y avícolas. Agron. Costarricense 27(1):79-89.

Hancock, D.W. 2011. Using relative forage quality to categorize hay. University of Georgia, GA, USA.

Harper, K.J., and D.M. McNeill. 2015. The role iNDF in the regulation of feed intake and the importance of its assessment in subtropical ruminant systems (the role of iNDF in the regulation of forage intake). Agriculture 5:778-790. doi:10.3390/ agriculture 5030778

Hiriart, M. 2008. Ensilados. Procesamiento y Calidad. $2^{\text {da }}$ ed. Editorial Trillas, MEX.

Hoffman P. 2005. Ash content of forages. Focus on Forages 7(1):1-2.

Jung, H.G. 2012. Forage digestibility: the intersection of cell wall lignification and plant tissue anatomy. University of Florida, FL, USA. http://dairy.ifas.ufl.edu/rns/2012/12JungRNS2012.pdf (consultado 17 feb. 2017).

Kung, L., and R. Shaver. 2001. Interpretation and use of silage fermentation analysis reports. Focus on forage 3(13):1-5.

Licitra, G., T.M. Hernandez, and P.J. Van-Soest. 1996. Standardization of procedures for nitrogen fractionation of ruminant feeds. Anim. Feed Sci. Technol. 57:347-358. doi:10.1016/0377-8401(95)00837-3

Lima-Orozco, R., A. Castro-Alegría, and V. Fievez. 2013. Ensiled sorghum and soybean as ruminant feed in the tropics, with emphasis on Cuba. Grass Forage Sci. 68:20-32. doi:10.1111/j.1365-2494.2012.00890.x

López-Herrera, M., y E. Briceño-Arguedas. 2017. Efecto de la especie de leguminosa y la fuente de carbohidratos en la calidad física y química de mezclas para ensilaje. Nutr. Anim. Trop. 11(1):52-73. doi:10.15517/nat.v11i1.29605

López-Herrera, M., A. Rojas-Bourrillon, y C. Zumbado-Ramírez. 2017. Características nutricionales y fermentativas de ensilados de pasto Camerún con plátano Pelipita. Agron. Mesoam. 28:3 doi:10.15517/ma.v28i3.25237

López-Herrera-Herrera, M., R. WingChing, y A. Rojas-Bourrillón. 2009. Características fermentativas y nutricionales del ensilaje de rastrojo de piña (Ananas comosus). Agron. Costarricense 33:1-15.

López-Herrera, M., R. WingChing-Jones, y A. Rojas-Bourrillon. 2015. Valoración nutricional de ensilajes de corona de piña con adición de heno y urea. Nutr. Anim. Trop. 9(2):65-90. doi:10.15517/nat.v9i2.21605 
McDermott, J.J., S.J. Staal, H.A. Freeman, M. Herrero, and J.A. Van-de-Steeg. 2010. Sustaining intensification of smallholder livestock systems in the tropics. Livest. Sci. 130:95-109. doi: 10.1016/j.livsci.2010.02.014

McDonald, P. 1981. The biochemistry of silage. John Wiley, NY, USA.

Mohapatra, D., S. Mishra, and N. Sutar. 2010. Banana and its by-product utilization: an overview. J. Sci. Ind. Res. 69:323-329.

Montero, E. 2016. Evaluación de las propiedades fermentativas, nutricionales y el costo de elaboración de ensilajes de Poró (Erythrina sp) y Cratylia (Cratylia argentea) con niveles crecientes de inclusión de guineo cuadrado (Musa sp), para alimentación de ruminates. Tesis Lic., Universidad de Costa Rica, San José, CRC.

Mühlbach, P.R. 2005. Uso de aditivos para mejorar el ensilaje de los forrajes tropicales. Universidad Federal de Río Grande del Sur, Porto Alegre, BRA.

Neumann, M., R. Oliboni, M.R. Oliveira, M.V. Faria, R.K. Ueno, L.L. Reinerh, and T. Durman. 2010. Chemicals additive used in silages. Appl. Res. Agrotechol. 3(2):187-208. doi:10.5777/paet.v3i2.1155

Neureiter, M., T. Pereira-dos-Santos, C.P. Lopez, H. Pichler, R. Kirchmayr, and R. Braun. 2005. Effect of silage preparation on methane yields from whole crop maize silages. In: B.K. Ahring, and H. Hartmann, editors, Proceedings of the 4th International Symposium on Anaerobic Digestion of Solid Waste. BioCentrum-DTU, Copenhagen, DEN. p. 109-115.

NRC (National Research Council). 2001. Nutrient requirements of dairy cattle. $7^{\text {th }}$ ed. National Academy Press, WA, USA.

Pardo, O., J.E. Carulla, y H.D. Hess. 2008. Efecto de la relación proteína y energía sobre los niveles de amonio ruminal y nitrógeno ureico en sangre y leche, de vacas doble propósito del piedemonte llanero, Colombia. Rev. Colom. Cienc. Pecu. 21:387-397.

Pelissari, F.M., M.M. Andrade-Mahecha, P.J. Do-Amaral-Sobral, and F.C. Menegalli. 2012. Isolation and characterization of the flour and starch of plantain bananas (Musa paradisiaca). Starch 64:382-391. doi:10.1002/star.201100133

Quiceno, M.C., G.A. Giraldo, y R.H. Villamizar. 2014. Caracterización fisicoquímica del plátano (Musa paradisiaca sp. AAB, Simmonds) para la industrialización. UGciencia 20:48-54.

Ramírez, R., R.G. Ramírez, y F. López. 2002. Factores estructurales de la pared celular del forraje que afectan su digestibilidad. Cienc. UANL 2:180-189.

Ravi, I., and M.M. Mustaffa. 2013. Starch and amylose variability in banana cultivars. Ind. J. Plant Physiol. 18:83-87. doi:10.1007/s40502-013-0014-2

Teixeira, J.C., e G.A Andrade. 2001. Carboidratos na alimentação de ruminantes. Em: A.R. Evangelista, et al., editores, Memoria II Simpósio de Forragicultura e Pastagens, Lavras. Editora UFLA, Lavras, BRA. p. 165-210.

Tobía, C. 2004. Introducción del ensilaje de soya en un sistema de producción intensiva de leche en el trópico húmedo de Costa Rica. Tesis Ph.D., Universidad de Costa Rica, San José, CRC.

Van-Soest, P.V., J.B. Robertson, and B.A. Lewis. 1991. Methods for dietary fiber, neutral detergent fiber, and nonstarch polysaccharides in relation to animal nutrition. J. Dairy Sci. 74:3583-3597. doi:10.3168/jds.S0022-0302(91)78551-2

Vargas-Rodríguez, C.F. 2005. Valoración nutricional y degradabilidad ruminal de genotipos de sorgo forrajero (Sorghum sp). Agron. Mesoam. 16:215-223. doi:10.15517/am.v16i2.11874

Villalobos, L., y J. Arce. 2014. Evaluación agronómica y nutricional del pasto estrella africana (Cynodon nlemfuensis) En la zona de Monteverde, Puntarenas, Costa Rica. II. Valor Nutricional. Agron. Costarricense 38:133-145.

Villalobos, L., y J.M. Sánchez. 2010. Evaluación agronómica y nutricional del pasto ryegrass perenne tetraploide (Lolium perenne) producido en lecherías de las zonas altas de Costa Rica. II. Valor Nutricional. Agron. Costarricense 34:43-52. 
Wadhwa, M., and M.P.S. Bakshi. 2013. Utilization of fruit and vegetable wastes as livestock feed and as substrates for generation of other value-added products. FAO, Rome, ITA. http://www.fao.org/docrep/018/i3273e/i3273e.pdf (accessed 9 Aug. 2017).

Weiss, W.P. 2004. Fine-tuning energy calculations. In: M. Eastridge, editor, Proceedings of the Tri-State Dairy Nutrition Conference. Purdue University, Michigan State University, and Ohio State University, Fort Wayne, IN, USA. p. 131-142.

WingChing, R., y G. Alvarado. 2009. Valor nutricional del heno de Transvala inoculado con el hongo Pleurotus ostreatus sp. Agron. Costarricense 33:147-153.

Yitbarek, M.B., and B. Tamir. 2014. Silage additives: Review. Open J. Appl. Sci. 4:258-274. doi:10.4236/ojapps.2014.45026

Zebeli, Q., D. Mansmann, H. Steingass, and B.N. Ametaj. 2010. Balancing diets for physically effective fibre and ruminally degradable starch: A key to lower the risk of sub-acute rumen acidosis and improve productivity of dairy cattle. Livest. Sci. 127:1-10. doi:10.1016/j.livsci.2009.09.003 